\title{
Evolución temporal del área de los pinares del Valle de Lozoya
}

\section{Temporal evolution in the pine forests of Valle de Lozoya}

\author{
Jesús Rascón ${ }^{1 *} \mathbb{D}$, Fernando Corroto ${ }^{1}$
}

\section{RESUMEN}

Uno de los lugares más emblemáticos de la Comunidad de Madrid es el Valle del Lozoya. Este lugar ha padecido abandono y maltrato por el exceso de explotación y a causa de las inadecuadas reforestaciones que comenzaron a realizarse a finales del siglo XIX. Fue en esta época en la que el interés por el entorno hace que se comience a legislar, procurando la preservación y, sobre todo, el aumento de la masa arbórea de la zona, con un resultado a veces dudoso. Con este estudio, se pretende explicar de modo conciso la evolución de los pinares de este valle madrileño. Para ello, se han trazado mapas, completándolos con tablas, y como resultado se puede observar el crecimiento de las áreas, gracias a la aplicación de las diferentes leyes y planes forestales, tales como el Plan General de Repoblación Forestal de España o el Plan Forestal de la Comunidad de Madrid. Las figuras de protección como el Parque Natural de Peñalara y el Valle del Paular también han ayudado al crecimiento de las áreas.

Palabras claves: Sierra de Guadarrama, Rio Lozoya, Pinus sylvestris, Pinus pinaster, repoblaciones.

\begin{abstract}
One of the places most emblematic of the Community of Madrid is Lozoya Valley. This place has suffered neglect and abuse by over-exploitation and because of inadequate reforestation that began performing in the late s. XIX. It was at this time when interest in the environment makes you start to legislate ensuring the preservation and especially the increasing tree mass in the area, with sometimes dubious results. This study aims to explain in a concise manner the evolution of the pine forests of this Madrid valley for it have been drawing maps, supplementing with tables, and as a result you can see the growth areas, thanks to the application of different laws and forest plans as the Plan General de Repoblación de España or the Plan Forestal de la Comunidad de Madrid. Figures of protection as the Parque Natural de Peñalara y el Valle del Paular, have also helped the growth areas.
\end{abstract}

Keywords: Sierra de Guadarrama, Rio Lozoya, Pinus sylvestris, Pinus pinaster, reforestation. 


\section{INTRODUCCIÓN}

El valle del Lozoya es el valle más extenso de la Comunidad de Madrid, localizado en la zona norte, en la vertiente madrileña de la Sierra de Guadarrama. Este valle se forma del río Lozoya, que emerge en las cumbres más altas de la Sierra de Guadarrama; recorriendo, transversalmente, el norte de la Comunidad de Madrid hasta su desembocadura en el río Jarama. Las aguas del río Lozoya han abastecido, desde antaño, a las poblaciones cercanas; siendo, así mismo, fundamental para el suministro de agua en la ciudad de Madrid. A lo largo de su recorrido se han construido embalses que garanticen este suministro (Alcolea y García, 2006).

Históricamente, el Valle de Lozoya ha sido un lugar predilecto para la comunidad científica, lo que ha ayudado a que en el entorno del Valle surjan figuras de protección ambiental, tales como la cumbre, el circo y las lagunas de Peñalara (2 430 m.s.n.m.). Además, encontramos las Zonas de Especial Protección para las Aves (ZEPA), y es que en el valle se encuentra la ZEPA del Alto de Lozoya. Otras figuras de protección son los Lugares de interés comunitario (LIC); por ejemplo, el LIC de la Cuenca del Río Lozoya y la Sierra Norte. Por último, los embalses y humedales del valle de Lozoya están protegidos por la Ley 7/1990 de la Comunidad de Madrid (Aceituno-Mata, 2010). Con la reciente declaración del Parque Nacional de la Sierra de Guadarrama, se han elevado los niveles de protección de estas zonas, con las figuras de protección generadas por este proyecto (BOE, 2013).

La vegetación del Valle tiene una gran diversidad, gracias a los múltiples ambientes que confluyen en él, ya que las notables diferencias de altitud generan variaciones climáticas que determinan las formaciones vegetales de cada zona. De todo, podemos destacar los pinares de pino albar (Pinus sylvestris) y las dehesas de encinas (Quercus ilex subsp. ballota). No obstante, gran parte de las masas arbóreas han estado influidas por los usos dados por las poblaciones a lo largo del tiempo (agricultura, ganadería, aprovechamiento de leña para combustible o madera, etc.), los que en algunos casos han llevado a la casi desaparición de varias especies arbóreas de la zona como el roble albar (Quercus petrea). Sin embargo, gran parte de estos usos han ido disminuyendo a lo largo del siglo XX (Iriarte, 2003; CMAVOTCM, 2010).

Los pinares, objeto de este estudio, tanto los de origen natural como los resultantes de la repoblación, sobre todo los de Pinus sylvestris y Pinus pinaster, han sido aprovechados ordenadamente desde hace más de un siglo, lo que ha favorecido su extensión hacia zonas de menor altitud. Un buen ejemplo de ello es el pinar de "Los Belgas", en Rascafría, de origen natural, que es gestionado desde hace más de 160 años por propiedad privada. También habría que destacar que este pinar está dentro de la ZEPA del Alto de Lozoya, debido a que en él se encuentran algo más de 57 nidos de buitre negro (Aegypius monachus) (CMAVOTCM, 2008; Bravo, 2009).

No obstante, la mayoría de la superficie de los pinares del valle de Lozoya provine de repoblaciones. Estas vienen dadas porque España ha sufrido, a lo largo de su historia, un gran proceso de deforestación, que también afectó al Valle de Lozoya. Esta deforestación, dejó una gran superficie forestal muy degradada, quedando muy pocos territorios en buenas condiciones en 1860 (Montero y Serrada, 2013). Las causas que propiciaron esta deforestación son: el aprovechamiento abusivo de madera y leña, las rotaciones de terrenos forestales para cultivos agrícolas, el pastoreo excesivo por la carga, y los incendios forestales (Bravo, 2009). A mediados del siglo XIX se aprecia una sensibilidad frente a la deforestación, a causa de que en esta época se redactan los primeros documentos de carácter legislativo en los que se menciona la necesidad de repoblar terrenos afectados por incendios, o por las rotaciones de los cultivos o pastos. No obstante, la materialización de estas primeras leyes fue muy escasa (Iglesias, 2010). Uno de los primeros documentos para luchar contra la deforestación fue la Ley sobre Repoblación, Fomento y Mejora de los Montes Públicos de 1877, cuyo objetivo era la protección, pero obtuvo un resultado muy pobre. A este le siguieron otros como el Plan sistemático de repoblación de cabeceras de cuencas hidrográficas de 1988 (Serrada, 2000), en el que la Comisión de Repo- 
blación, para realizar las repoblaciones, recomendó el uso de pino silvestre o albar (Pinus sylvestris) entre 1400 y $1800 \mathrm{~m}$ de altitud, por debajo de estas altitudes recomendó el melojo (Quercus pyrenaica), aunque en las zonas donde esté desaparecía, se optó por el pino negral (Pinus pinaster). Por último, para las zonas por encima del pino silvestre o albar se propuso curiosamente repoblar con abeto (Abies alba) y pino negro (Pinus uncinata) por su madera de gran calidad y su resistencia al frío (López-Estebanez y Saéz-Pombo, 2003). No obstante, el crecimiento de la superficie repoblada no fue excesivo; debido, en parte, a los escasos recursos que se pusieron a disposición en ese momento (Sanjuanbendito, 2011).

Durante la primera parte del siglo XX, se crean las Confederaciones Hidrográficas, lo que dio un nuevo empujón al Plan Nacional de Repoblaciones de los Montes y el Plan Nacional de Obras Hidráulicas. En 1939, una vez acabada la Guerra Civil, el estado empezó a idear planes, y a legislar con el fin de dar trabajo a la gran cantidad de desempleados que quedaron tras la Guerra Civil (Sanjuanbendito, 2011). Uno de los planes que se desarrollaron fue el Plan General de Repoblación Forestal de España (PGRFE), el que constaba de dos fases de repoblación. Para la primera se usaron especies frugales, debido a su capacidad de adaptación a terrenos degradados; y una segunda fase, que no se pudo llevar a cabo en su totalidad, en la que se usaron especies más exigentes, sobre las masas creadas (Iglesias, 2010). En el Valle de Lozoya, las repoblaciones de este plan tuvieron un gran impulso. La mayoría de los pinares que se repoblaron en esta época son los que se encuentran actualmente en las sierras de Madrid, que tras 70 años han formado grandes masas arboladas con una gran diversidad biológica.

Ya en la segunda parte del siglo XX, a partir de 1986, se percibe una disminución de las repoblaciones, debida, en parte, a la transferencia de los asuntos forestales a las Comunidades Autónomas, que en esos momentos no tenían la estructura administrativa y legislativa necesaria para llevar a cabo estas políticas repobladoras de las que, con anterioridad, se hacía cargo el Estado (Iglesias,
2009). Sin embargo, a principios de los noventa, las administraciones de las Comunidades Autónomas, se fueron adaptando para afrontar la política repobladora necesaria en cada uno de sus territorios (Serrada, 2000). La Comunidad de Madrid elaboró su Plan Forestal gracias a la Ley Forestal y de Protección de la Naturaleza de la Comunidad de Madrid de 1995. Dentro de este Plan se establecen los planes para la repoblación y la restauración de las cubiertas vegetales, tanto de carácter productivo como de carácter protector. Para cumplir este Decreto, se aprobó el Plan Forestal de la Comunidad de Madrid (PFCM) para el periodo 20002019 , en el que se declara como uno de los objetivos más importantes la defensa de los recursos hídricos y del suelo, evitando la erosión mediante repoblaciones. En 2006, se dio un nuevo impulso a este Plan Forestal, cuando se elabora un nuevo Plan de Repoblaciones para un periodo de cinco años (2006-2010). El Plan también contempla el mantenimiento de las masas naturales, ya sea para uso productivo o para su mejora ecológica y estructural, como en el caso de los Pinares de Pinus sylvestris de origen natural, que hay en el Valle de Lozoya (Sanjuanbendito, 2011).

Con la reciente declaración de Parque Nacional de la Sierra de Guadarrama, las zonas afectadas por este han tenido que modificar parte de las directrices y objetivos, que se contemplan en el Plan Forestal de la Comunidad de Madrid para el periodo 2000-2019, imponiendo unos objetivos nuevos, como por ejemplo la naturalización y restauración ambiental de las repoblaciones, o la prohibición de técnicas de repoblación como la de terrazas, que degradan, paisajística y ecológicamente, la zona o el uso de especies alóctonas (BOE, 2013).

Todo esto, seguramente ha afectado la distribución y extensión de los pinares tanto de origen natural, como producto de las repoblaciones a lo largo del último siglo. Dado que este estudio tiene como objetivo la aproximación al conocimiento de la evolución temporal de los pinares en el Valle de Lozoya, se va a evaluar cómo la legislación estatal (hasta 1986) y autonómica (desde 1986) han afectado a estos pinares del Valle de Lozoya. Para ello, se presentará una cartografía con mapas de 
distribución de los diferentes pinares, desde 1957 hasta 2011, respaldada con las correspondientes áreas de los pinares, que permitan reconstruir la evolución del área de extensión ocupada por los mismos, y la incidencia que sobre estos han tenido los cambios sociales y políticos que ha sufrido la zona en el último siglo.

\section{MATERIALY MÉTODOS}

El área de estudio que se aborda en esta investigación se sitúa en el sector Norte de la Comunidad de Madrid, correspondiente al valle formado por el río Lozoya. El Valle de Lozoya está constituido por 25 municipios, con una extensión de unos $775 \mathrm{~km}^{2}$. El punto más alto se sitúa en la cumbre de Peñalara a 2430 m.s.n.m. y el punto más bajo se encuentra en el municipio del Berruco a 933 m.s.n.m. Limita al Sur con la Cuerda Larga y la Sierra de la Morcuera, al Noreste con la Sierra de Guadarrama y al Este con la Sierra del Rincón (Figura 1).

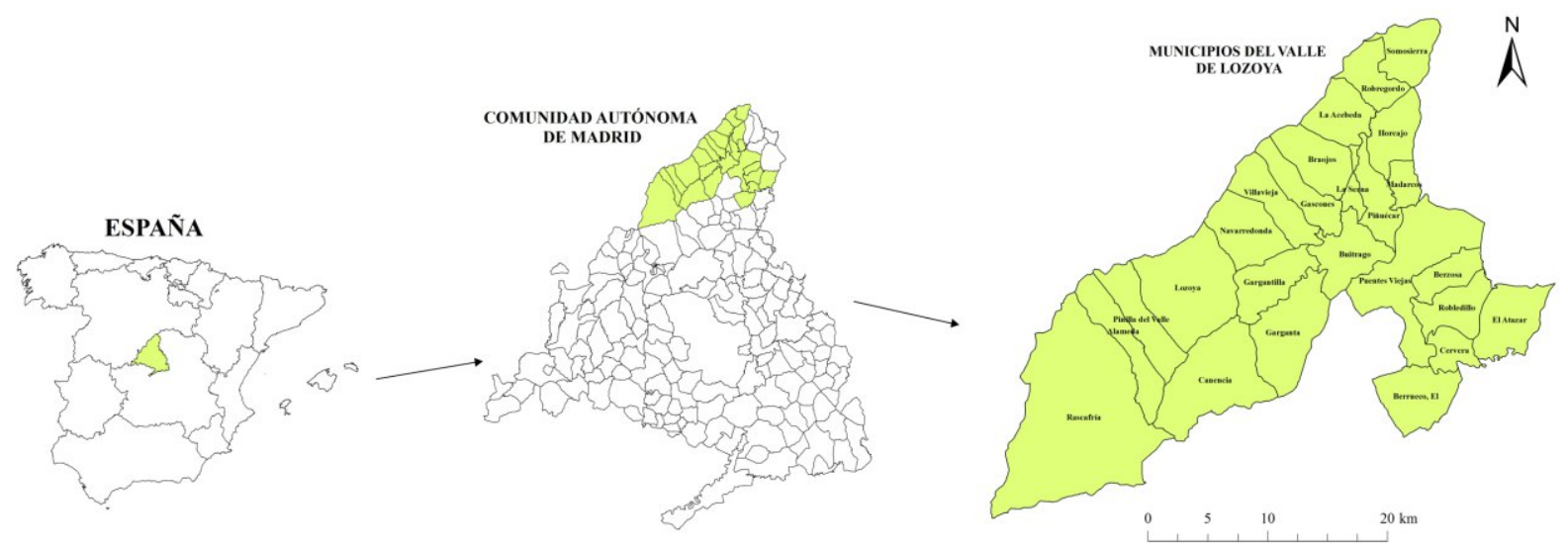

Figura 1. Mapa de ubicación de la zona de estudio, el Valle de Lozoya, en la Comunidad Autónoma de Madrid, España.

Para llevar a cabo el estudio, se revisaron y estudiaron fuentes bibliográficas: artículos científicos, libros, leyes, etc. También se consultaron mapas, ortofotos y visores cartográficos online, además de obtener capas en formato Shapefile: Mapa provincial de Madrid a escala 1:200.000 (IGN, 2014). Mapa forestal de España de 1966 a escala 1:400.000 (MAGRAMA, 2014). Mapa forestal de España de 1997 a escala 1:200.000: Hoja 5-5, correspondiente a Segovia (MAGRAMA, 2014). Mapa forestal de España de 2005 a escala 1:50.000: Hoja 28 correspondiente a la Comunidad de Madrid (MAGRAMA, 2014). Ortofotos de 2004 de la zona de estudio, a escala 1:50.000: hojas 458, 459, 483, 484, 485, 508, 509, 510 (IGN, 2014). Visor cartográfico de la Comunidad de Madrid, donde se observaron sobre todo las ortofotos de 1956 y 2011 y las imágenes por satélite de 1984 (CM, 20104). Capas en formato Shapefile de los ríos y embalses de España (MAGRAMA, 2014). Capas en formato Shapefile de los municipios de la Comunidad de Madrid (CM, 2014).

Los datos de las áreas de pinares del Valle de Lozoya, se obtuvieron a partir de mapas elaborados personalmen- te, debido a que los diferentes censos encontrados no aportan los datos de los años propuestos en cada mapa. Con todo el material recogido se han elaborado tres mapas de los pinares y las zonas de mezcla con frondosas, a través de los programas informáticos ArcGIS 10.1 y Adobe Photoshop Elements 7.0, de los últimos 60 años $(1956,1984,2011)$ para establecer, de forma visual, la evolución de estas áreas de pinares. A partir de estos mapas, se obtuvieron las áreas de extensión de los pinares, según su especie, en los años estudiados. Con estos datos se han hecho tablas y se han analizado mediante Excel, para calcular el porcentaje de crecimiento o decrecimiento que han tenido dichas áreas de pinares en el Valle de Lozoya.

\section{RESULTADOS}

En los siguientes mapas (Figuras 2 a 4), se ve la evolución de los pinares del Valle de Lozoya durante los últimos 60 años, indicando con diferentes colores los pinares, según el origen de estos (natural o de repoblación) y de la especie que los forma. También se han señalado algunos de los picos y Sierras ubicados en el Valle de Lozoya. 


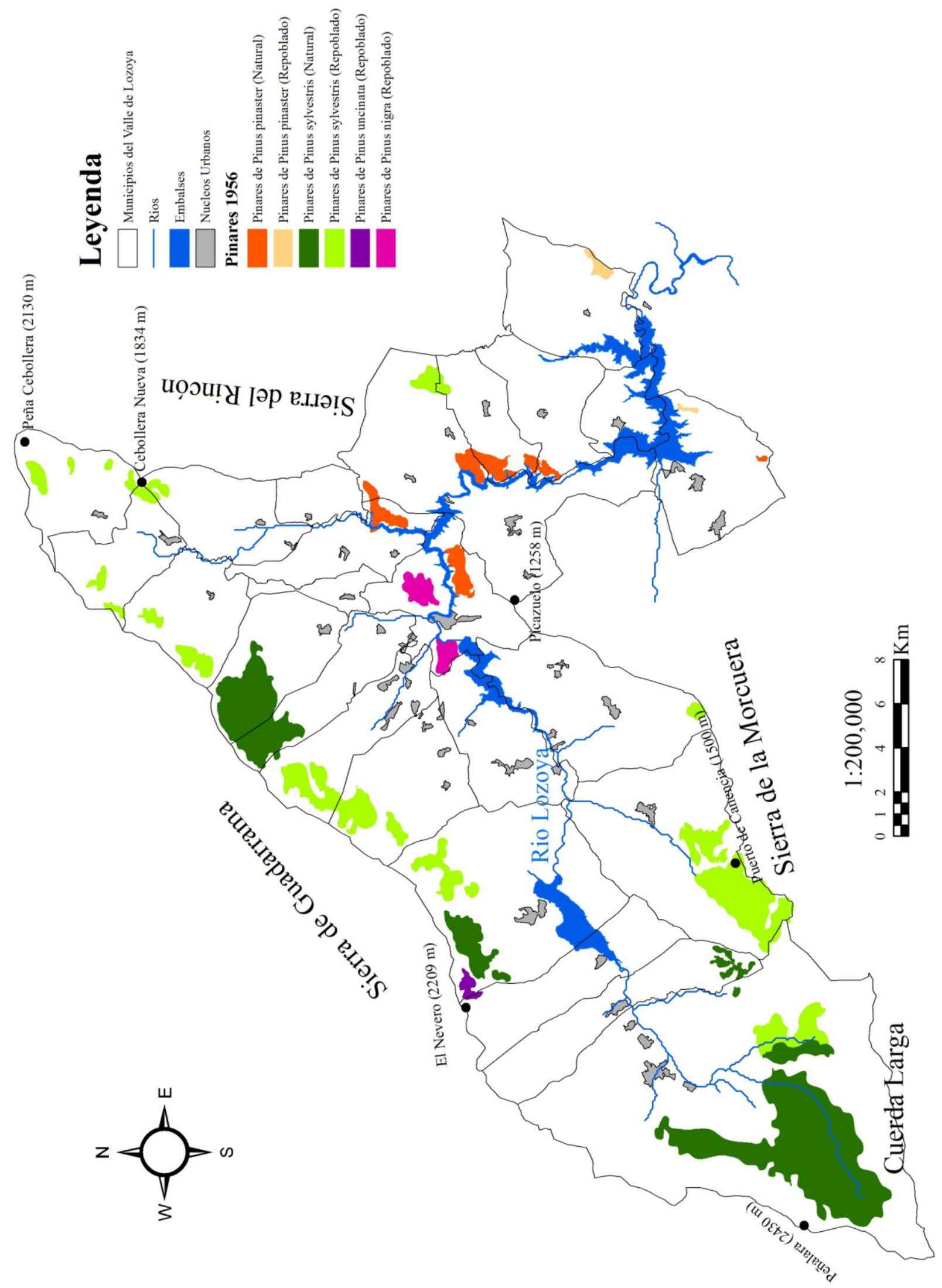

Figura 2. Mapa de los Pinares de Lozoya en 1956. 


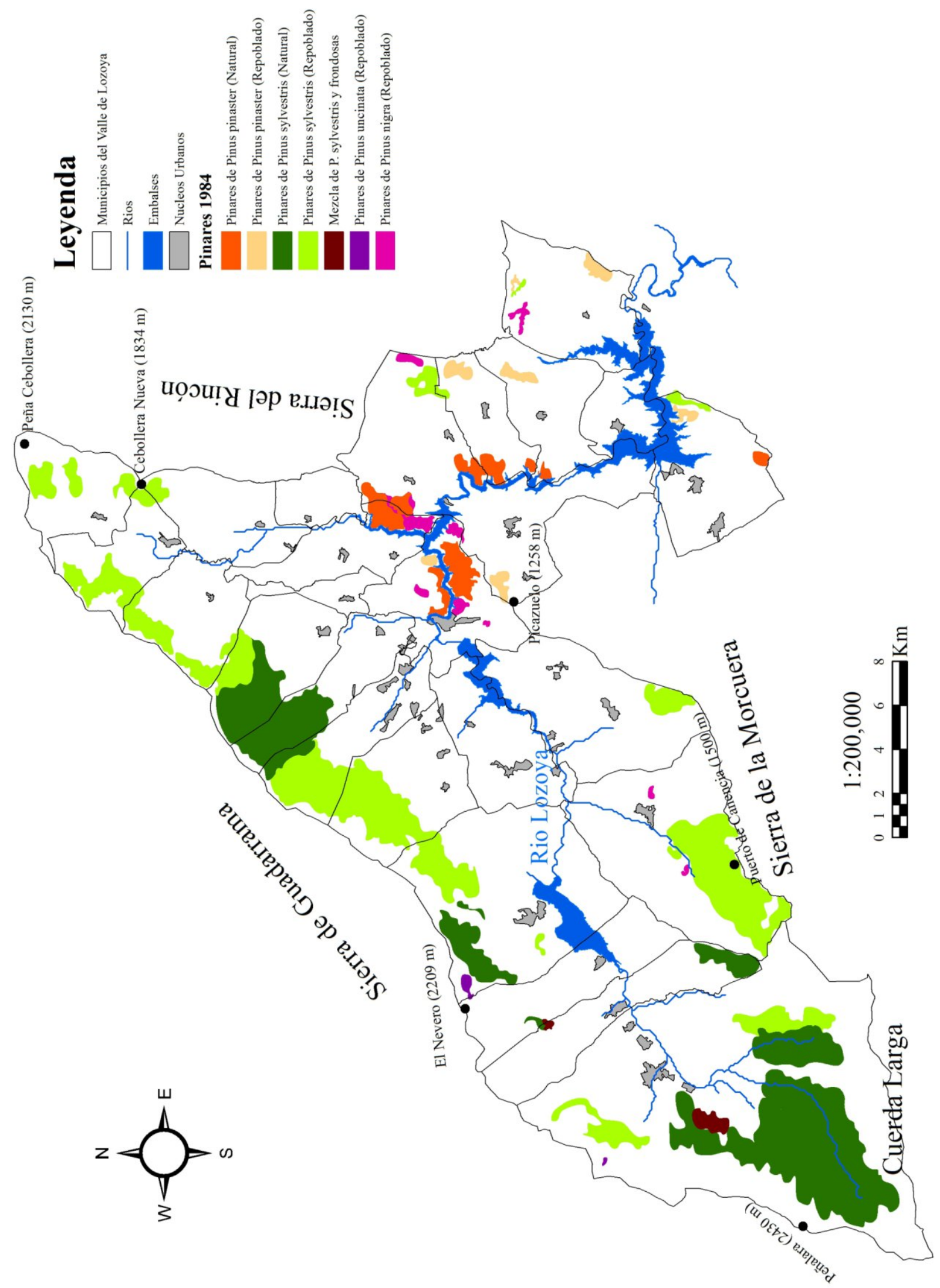

Figura 3. Mapa de los Pinares de Lozoya en 1984. 


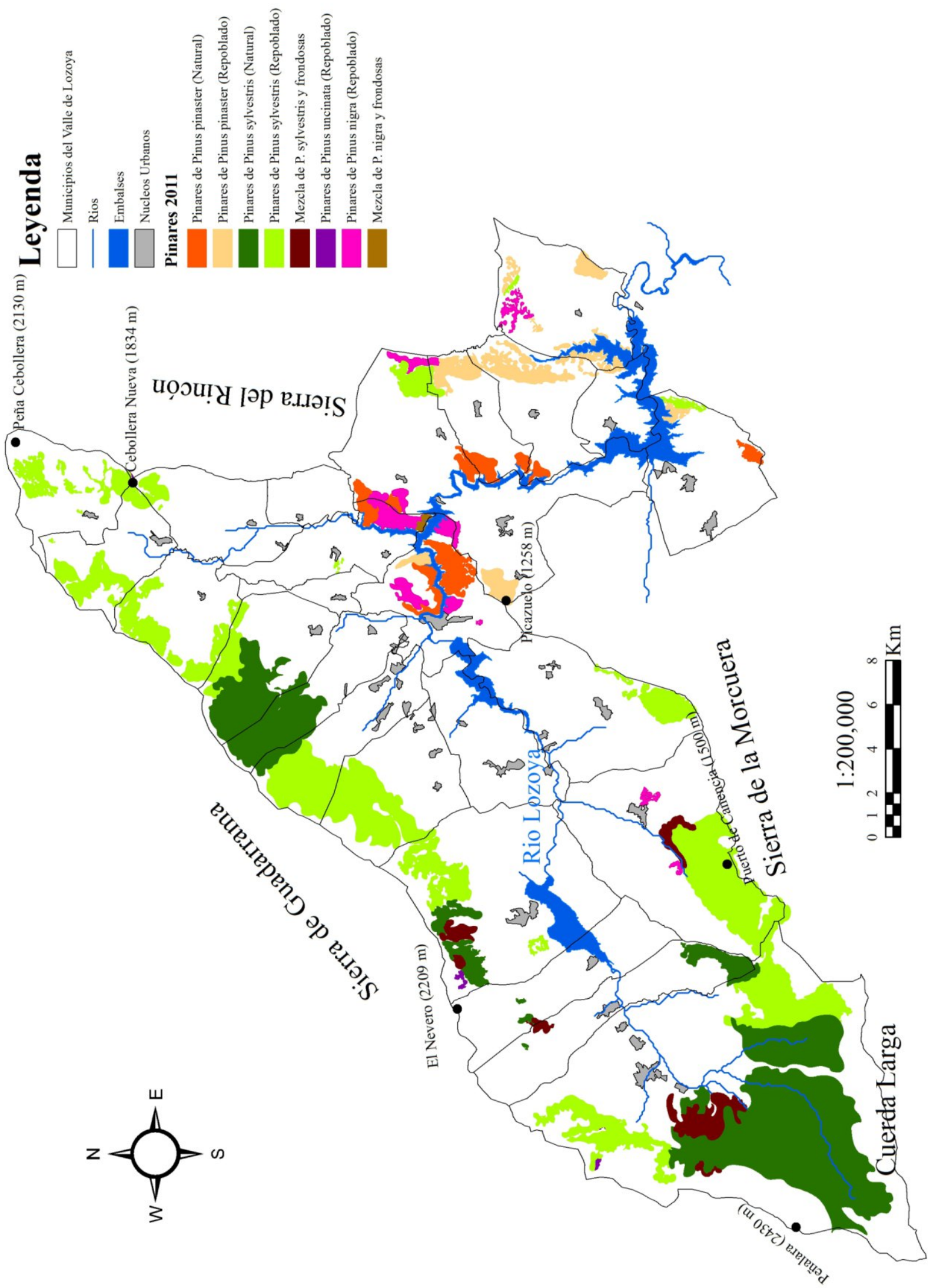

Figura 4. Mapa de los Pinares de Lozoya en 2011. 
En la Tabla 1 se muestran las áreas de los pinares, dependiendo del origen de estos (natural o de repoblación) y de la especie que los forma, en los años 1956, 1984 y 2011.

En esta tabla, cabe destacar que en 1956 podía encontrarse una mayor cantidad de hectáreas de P. sylvestris de origen natural, 4224 hectáreas frente las 3003 hectáreas cuyo origen es la repoblación. En la Tabla 2, se muestran los porcentajes de crecimiento de las áreas de los diferentes pinares durante los últimos 60 años: años 1956 - 1984 y $1984-2011$.

Tabla 1. Áreas de los diferentes pinares en 1956, en 1984 y en 2011

\begin{tabular}{|c|c|c|c|}
\hline Tipo de Pinar & Áreas 1956(ha) & Áreas 1984 (ha) & Áreas 2011 (ha) \\
\hline Mezcla de $P$. nigra y frondosas & 0 & 0 & 29 \\
\hline Mezcla de $P$. sylvestris y frondosas & 0 & 136 & 551 \\
\hline Pinares de $P$. nigra $(\mathrm{R})$ & 269 & 325 & 692 \\
\hline Pinares de P.pinaster (N) & 616 & 857 & 882 \\
\hline Pinares de P.pinaster (R) & 91 & 374 & 1095 \\
\hline Pinares de P.sylvestris (N) & 4224 & 5491 & 6778 \\
\hline Pinares de P.sylvestris (R) & 3003 & 6318 & 8289 \\
\hline Pinares de $P$. uncinata (R) & 60 & 38 & 48 \\
\hline Total de Pinares Naturales & 4840 & 6348 & 7660 \\
\hline Total de Pinares Repoblados & 3423 & 7055 & 10124 \\
\hline
\end{tabular}

Tabla 2. Crecimiento de las áreas de los pinares (1956-1984 y 1984-2011)

\begin{tabular}{|c|c|c|}
\hline Tipo de Pinar & Crecimiento(1956-1984) & Crecimiento(1984-2011) \\
\hline Mezcla de $P$. nigra y frondosas & $0,00 \%$ & $100,00 \%$ \\
\hline Mezcla de P. sylvestris y frondosas & $100,00 \%$ & $305,15 \%$ \\
\hline Pinares de $P$.nigra (R) & $20,82 \%$ & $112,92 \%$ \\
\hline Pinares de P.pinaster (N) & $39,12 \%$ & $2,92 \%$ \\
\hline Pinares de P.pinaster (R) & $310,99 \%$ & $192,78 \%$ \\
\hline Pinares de P.sylvestris (N) & $30,00 \%$ & $23,44 \%$ \\
\hline Pinares de P.sylvestris (R) & $110,39 \%$ & $31,20 \%$ \\
\hline Pinares de $P$. uncinata (R) & $-36,67 \%$ & $26,32 \%$ \\
\hline Total de Pinares Naturales & $31,16 \%$ & $20,67 \%$ \\
\hline Total de Pinares Repoblados & $106,11 \%$ & $43,50 \%$ \\
\hline
\end{tabular}

En esta tabla destacan los pinares de $P$. uncinata, y es que el primero decrece su área total $(-36,67 \%)$ y después crece $(26,32 \%)$. También hay que mencionar los perio- dos de crecimiento de $100 \%$, esto se debe a que se pasó de un año en el que no había ninguna hectárea de masa arbórea a otro en el que esta comienza a constatarse.

Tabla 3. Tabla del crecimiento total de las áreas de los pinares

\begin{tabular}{|c|c|}
\hline Tipo de Pinares & Crecimiento en los últimos 60 años \\
\hline Mezcla de $P$. nigra y frondosas & $100,00 \%$ \\
\hline Mezcla de P. sylvestris y frondosas & $100,00 \%$ \\
\hline Pinares de $P$.nigra (R) & $157,25 \%$ \\
\hline Pinares de P.pinaster (N) & $43,18 \%$ \\
\hline Pinares de P.pinaster (R) & $1103,30 \%$ \\
\hline Pinares de P.sylvestris (N) & $60,46 \%$ \\
\hline Pinares de P. sylvestris (R) & $176,02 \%$ \\
\hline Pinares de P. uncinata (R) & $-20,00 \%$ \\
\hline Total de Pinares Naturales & $58,26 \%$ \\
\hline Total de Pinares Repoblados & $195,76 \%$ \\
\hline
\end{tabular}


En la Tabla 3, destaca el decrecimiento de los pinares de $P$. uncinata (-20\%), pero sobre todo el gran crecimiento de los pinares de P. pinaster (1103, $3 \%)$.

\section{IV.DISCUSIÓN}

En la evolución de las áreas de los pinares en el Valle de Lozoya, podemos apreciar, al ver los mapas de los pinares de 1956, 1984 y 2011, que su área ha tenido un crecimiento a lo largo de los últimos 60 años, tanto los de origen de repoblación como los de origen natural. Sobre todo, los pinares que han tenido un mayor crecimiento son los de origen de repoblación, con un crecimiento de su área del 195,76\%. Mientras tanto, las áreas de pinar de origen natural han tenido un crecimiento del $58,26 \%$, gracias al mantenimiento de estos pinares naturales, dándoles un uso productivo en su mayor parte. Al observar la Tabla 1 se observa que en 1956 había más área de pinar origen natural (4840 hectáreas) que de origen de repoblación (3423 hectáreas). Sin embargo con todas las políticas forestales aplicadas, en concreto el PGRFE y el PFCM, han provocado que se dé la situación inversa, ya que en 2011 hay más pinar de origen de repoblación (10 124 hectáreas), que de origen natural (7660 hectáreas).

Los pinares que más área ocupan en el Valle de Lozoya son los de P. sylvestris, tanto los naturales como los repoblados (Tabla 1). Sin embargo, los pinares naturales de $P$. sylvestris, han crecido un $60,46 \%$, en los últimos 60 años, frente al 176,02\% de crecimiento que han tenido los pinares de repoblación de la misma especie (Tabla 3).

El gran crecimiento de los pinares de repoblación de $P$. sylvestris se deben, en parte, a que es una de las especies que más se usó y recomendó para las repoblaciones en la zona por parte de la Comisión Repobladora de la Cuenca del Lozoya y el PGRFE, sobre todo por su carácter productor, aunque también en parte por su carácter protector, además de ser autóctona de la zona. Un gran ejemplo de ello es el pinar de Canencia que es una de las repoblaciones más antiguas de la zona, que se repobló para proteger el suelo. Algunos autores lo consideran natural porque se ha integrado ecológica- mente y estructuralmente a la zona. Cabe destacar que las áreas de pinar repoblado de $P$. sylvestris crecieron más entre 1956 y 1984 , un $110,36 \%$, que entre 1984 y 2011, un 31,20\%. Esto se debe a que a partir de 1986 hubo un descenso de las repoblaciones al traspasar los asuntos forestales del Estado a las Comunidades Autónomas. Además de que se favoreció el uso de otras especies de pino, con un crecimiento más rápido, sobre todo en las repoblaciones desarrolladas por el PGRFE (González-Pellejero y Álvarez-Cañada, 2004).

En cuanto a los pinares de $P$. sylvestris de origen natural, han tenido un buen crecimiento de sus áreas, gracias a la creación de figuras de protección y a las políticas forestales aplicadas, que gestionaban y gestionan su mantenimiento y uso. Debido a que en parte se le dio un uso productivo, como los del Valle de Paular, donde se encuentra el Bosque de los Belgas, de gestión privada. Mientras que otros como los pinares de Peñalara, se gestionaron por la protección de recursos hídricos de la zona, y es que en las Cumbres del Peñalara nace el río Lozoya. Por eso, estos pinares han tenido un crecimiento más o menos constante, ya que de 1954 a 1984 crecieron un 30\%, y entre 1984 y 2011 crecieron un $23,44 \%$ (Tabla 2 ).

Los pinares de P. pinaster, tanto los naturales como los repoblados han aumentado (Tabla 4). Aunque, los pinares naturales de $P$. pinaster han crecido un $43,18 \%$ en los últimos 60 años, frente al 1103,30\% de crecimiento que han tenido los pinares de repoblación de $P$. pinaster, siendo el crecimiento más alto que hemos observado (Tabla 3).

El gran crecimiento de los pinares de repoblación de $P$. pinaster se debe, en parte, a que es una especie que se recomendó para las repoblaciones en la zona (más que p. sylvestris), por su carácter productor, aunque también por su carácter protector, además de ser autóctona de la zona. La Comisión Repobladora de la Cuenca del Lozoya, lo sugiere para las repoblaciones, pero para las zonas donde no se podía repoblar con Quercus pyrenaica. Pero sobre todo fue recomendado por el PGRFE y lo podemos observar en los mapas de las Figuras 1 a 3, dado que en 1957, solo había un pequeño 
pinar de repoblación, que ha creció muy poco, en el municipio de El Atacar, mientras que en 1984, ya hay más pinares de un tamaño considerable. Un gran ejemplo de estos pinares de $P$. pinaster desarrollados por las repoblaciones del PGRFE, es el pinar de Picazuelas, en el municipio de Puentes Viejas, que es uno de los pinares de $P$. pinaster que más ha crecido en los últimos 60 años. Por tanto, el crecimiento de las áreas de pinar repoblado de $P$. pinaster fue mayor entre 1956 y 1984 (un 310,99 \%), que entre 1984 y 2011 (un $192,78 \%$ ). Por lo que podemos decir que había cierto favoritismo a desarrollar repoblaciones con esta especie, por parte del PGRFE (Serrada et al., 2008) y a que a partir de 1986 hubo un descenso de las repoblaciones, por traspasar los asuntos forestales del Estado a las Comunidades Autónomas.

En cuanto a los pinares de $P$. pinaster, de origen natural, han tenido un crecimiento moderado a bajo, parcialmente por la aplicación de políticas forestales para la gestión del mantenimiento y uso de estos pinares. De 1954 a 1984, crecieron un 39,12\% y entre 1984 y 2011 crecieron un 2,92\%. Principalmente se debe a que, en el primer periodo, el $P$. pinaster, tuvo un gran uso en la zona por la extracción de resina, lo que favoreció su crecimiento, el que se puede ver en los mapas de los pinares de 1956 y 1984 (Figuras 2 y 3), sobre todo el crecimiento que se da en los municipios de Buitrago y Piñuecar. Mientras que en el segundo periodo, este uso del $P$. pinaster se ha perdido, por lo que los pinares simplemente se han mantenido con un crecimiento natural, el cual es lento. Esto también se observa en los mapas de 1984 y 2011 (Figuras 3 y 4), más la Tabla 1, donde vemos que las áreas son casi las mismas.

Los pinares de P. nigra del Valle de Lozoya, al contrario que $P$. pinaster y $P$. sylvestris, son de origen de repoblación y también han crecido en los últimos 60 años, en concreto un 157,25\% (Tabla 3) (Gómez et al., 2007).

El gran crecimiento de los pinares de repoblación de $P$. nigra se debe, en parte, a que es una especie que se usó para las repoblaciones en la zona por su carácter pro- tector, aunque por la gran calidad de su madera, también se repobló con motivos productores. Fue una especie recomendada por la Comisión de Repoblación de la Cuenca del Lozoya y por el PGRFE, pero sobre todo por el PFCM, ya que protege los suelos. Esto lo podemos observar en los mapas de las Figuras 1 a 3, teniendo en cuenta que en 1957 había solo dos pinares en municipio de Buitrago, producto de repoblaciones anteriores al PGRFE; en 1984, estos pinares se ven reducidos en más de la mitad, seguramente por los usos productivos que les dieron en la zona, pero a su vez hay más zonas del Valle donde aparecen pequeños pinares de P. nigra gracias al PGRFE, como por ejemplo en el municipio de Piñuecar o en Canencia, seguramente para darles un uso productivo. Mientras en 2011, las masas observadas en 1984, han aumentado su área, debido en parte al PFCM, por su carácter protector. Por tanto, el crecimiento de las áreas de pinar repoblado de P. nigra fue mayor entre 1984 y 2011, un $112,92 \%$, que entre 1956 y 1984, un $20.82 \%$ (Tabla 2) por lo que podemos decir que había cierto interés por desarrollar repoblaciones con esta especie por parte del PGRFE y del PFCM, para proteger los suelos, sobre todo en zonas donde $P$. pinaster y $P$. sylvestris no se desarrollan (Mendoza y Olmo, 1992).

Por último, están los pinares de P. uncinata, que son los que menos área ocupan en el Valle de Lozoya (Tabla 1). Y al igual que $P$. nigra, la totalidad de estos pinares es de origen de repoblación sin embargo, son los únicos que han decrecido en los últimos 60 años, en concreto un $-20,00 \%$ (Tabla 3 ).

El decrecimiento de los pinares de repoblación de $P$. uncinata, se debe en parte a que es una especie alóctona, más bien típica de los Pirineos y los Alpes, por lo que las otras especies de pinos no dejan que se expanda, además de que sus requerimientos ecológicos son muy diferentes, como por ejemplo el necesitar grandes alturas, razón por la cual solo lo encontramos en los puntos más altos del Valle. Este decrecimiento, también puede deberse a que su crecimiento es lento, y su vida corta, por lo que las repoblaciones hechas con $P$. uncinata, si no se mantienen, se van perdiendo (Gó- 
mez y Mata, 1992). Por eso, podemos decir que las repoblaciones de $P$. uncinata, desarrolladas por la Comisión de Repoblación de la Cuenca del Lozoya, el PGRFE y el PFCM, han sido de carácter experimental, para proteger los suelos. Esto se puede ver al observar los mapas de 1956, 1984 y 2011 (Figuras 2 a 4), dado que en 1956 se ve un pinar cerca del pico de El Nevero, en el término municipal de Lozoya, seguramente uno de los pocos que quedaron de las repoblaciones experimentales hechas por la Comisión de Repoblación de la Cuenca del Lozoya. En 1984 este pinar decrece casi un 36,6\% (Tabla 2), aunque en el Municipio de Rascafría aparece un pequeñísimo pinar de $P$. uncinata, desarrollado por el PGRFE. Por último en 2011, observamos que estos dos pinares han crecido, además de que el PFCM desarrollo una pequeña repoblación de este pino cerca de la Cebollera Nueva, por lo que el área de pinar de P. uncinata creció un 26,32\% (Tabla 2).

\section{CONCLUSIONES}

Por tanto, se puede decir que los pinares, tanto naturales como de repoblación, han tenido un crecimiento de sus áreas, como vemos gracias a los datos obtenidos y analizados. Además cumplen algunos objetivos del actual PFCM, como por ejemplo los objetivos protectores o el objetivo de aumentar las masas arbóreas para que actúen como sumidero de carbono (fijación de CO2). También se cumplen los del PGRFE 19411986, como los de uso productivo y protectores del suelo, a causa de este plan se debe, gran parte del aumento de las áreas de pinares en el Valle de Lozoya como hemos visto.

Se puede concluir que la evolución temporal de las áreas de los pinares es más que apreciable, además de que esta evolución ha estado influida claramente por las diferentes políticas forestales que se han ido desarrollando, así como la creación de figuras como la ZEPA del Alto de Lozoya o el Parque Natural de Peñalara, que han conformado uno pinares con una alta valoración, no solo como recurso forestal, sino también como recurso histórico, cultural e incluso turístico.

\section{REFERENCIAS BIBLIOGRÁFICAS}

Aceituno-Mata, L. 2010. Estudio etnobotánico y agroecológico de la Sierra Norte de Madrid. Tesis de Doctorado. Universidad Autónoma de Madrid. Madrid(España).

Alcólea, M. A. y J. M. García. 2006. "El agua en la Comunidad de Madrid." Observatorio Medioambiental 9: 63-96

Bravo, E. 2009. "El Pinar de los belgas en El Paular: Más de 160 años de gestión privada del bosque". Revista Ambienta (87): 64-71

BOE (Boletín Oficial del Estado). 2013. Ley 7/2013, de 25 de junio de declaración del Parque Nacional de Sierra de Guadarrama. Ley Nacional. Madrid (España)

CM (Comunidad de Madrid). 2014. Mapas. http://www.comunidad.madrid/servicios/ma pas (Consultada el 02 de Agosto de 2014)

CMAVOTCM (Conserjería de Medio Ambientes, Vivienda y Ordenación del Territorio de la Comunidad de Madrid). 2008. PORN de la Sierra de Guadarrama en el ámbito territorial de la comunidad de Madrid. Madrid (España): Edición Comunidad de Madrid.

CMAVOTCM (Conserjería de Medio Ambientes, Vivienda y Ordenación del Territorio de la Comunidad de Madrid). 2010. Cuadernos del Puente del Perdón $N^{o}$ 2. Madrid (España): Edición Comunidad de Madrid.

Gómez, J. y R. Mata. 1992. “Actuaciones forestales publicas desde 1940. Objetivos, criterios y resultados". Agricultura y sociedad (65): 15 64

Gómez, J., G. Madrazo-García, y E. Sáez-Pombo. 2007 "Dinámica histórica de paisajes forestales y sostenibilidad". En Sextas Jornadas Cientificas del Parque natural de Peñalara y del Valle de El Paular. Rascafría (España).

González- Pellejero, R. y A. Álvarez-Cañada. 2004. "El Mapa Forestal de España, una obra secular (1868-1966) concluida por Luis Ceballos". Eria (64-65): $285-318$. 
Iglesias, A. 2010. "Apuntes sobre la situación de la repoblación forestal en España." Cuadernos De La Sociedad Española De Ciencias Forestales (31): 113-136

IGN (Instituto Geográfico Nacional). 2014. Cartografía y Datos geográficos. https://www.ign.es /web/cbg-area-cartografia (Consultada 02 de Agosto 2014)

Iriarte, I. 2003. “ La funcionalidad económica y social de los montes. Un esbozo de las transformaciones de largo plazo". Cuadernos De La Sociedad Española De Ciencias Forestales (16): 31-40

López-Estebanez, N. y E. Saéz-Pombo. 2003. “Análisis y valoración de los estudios de la Comisión Repoblación de la Cuenca del Lozoya, Madrid (1890 - 1895)". Cuadernos De La Sociedad Española De Ciencias Forestales (16): 255-260

MAGRAMA (Ministerio de Agricultura, Alimentación y Medioambiente). 2014. Datos Cartográficos. http://www.magrama.gob.es. (Consultada el 02 de Agosto de 2014)

Montero, G. y R. Serrada. 2013. La situación de los Bosques y el sector forestal en España. Pontevedra (España): Sociedad Española de Ciencias Forestales.

Sanjuanbendito, P. 2011. "La repoblación forestal en la Comunidad de Madrid". Foresta (52): 160-165

Serrada, R. 2000. Apuntes de Repoblaciones Forestales. Madrid (España): FUCOVASA.

Serrada, R., G. Montero, y J. A. Reque. 2008. Compendio de Selvicultura Aplicada en España. Madrid(España): FUCOVASA-INIA. 